ORIGINAL STUDY

\title{
The importance of computerized dynamic posturography in vestibular rehabilitation of patients with unilateral peripheral vestibular deficiency
}

\author{
Anamaria Andreia Ulmeanu', Andreea Didilescu ${ }^{2}$, Raluca Enache ${ }^{3}$, Gabriela Musat ${ }^{4}$, \\ Codrut Sarafoleanu ${ }^{3,4}$ \\ ${ }^{1}$ Bucharest Emergency Hospital, Romania
}

${ }^{2}$ Faculty of Dental Medicine, "Carol Davila” University of Medicine and Pharmacy, Bucharest, Romania

${ }^{3}$ ENT Sarafoleanu Medical Clinic, Bucharest, Romania

4"Carol Davila" University of Medicine and Pharmacy, Bucharest, Romania

\section{ABSTRACT}

OBJECTIVE. To evaluate the importance of computerized dynamic posturography in vestibular rehabilitation of patients with unilateral peripheral vestibular deficiency syndrome.

MATERIAL AND METHODS. The study was conducted on a group of 30 patients (33-78 years; mean age $( \pm$ SD) $=55.8 \pm 12.12)$ diagnosed with unilateral peripheral vestibular deficiency syndrome, which benefited from VR on a posturography platform. Assessment of the patients was made using the Sensory Organization Test before and after eight sessions of rehabilitation. We analyzed the results obtained with eyes open (EO) and eyes closed (EC) on static and foam platform. The following variables were evaluated: Romberg coefficient, statokinesigram (SKG), maximum amplitude of the degree of deviation in anterior/posterior and medial/lateral planes, SKG and the time interval of the game rehabilitation program.

RESULTS. The statistical analysis of the data revealed a strong correlation $(\mathrm{p}<0.05)$ for the studied parameters, especially when the test was performed with the eyes closed on foam platform. The analysis of the Romberg coefficient did not show statistically significant results $(\mathrm{p}>0.05)$ and the measured values were outside the range of normality even at the end of the rehabilitation program. SKG and the time interval reference of the game showed significant improvement of the parameters $(p<0.05)$; at the end of the rehabilitation sessions, $93.33 \%$ of the patients showed full recovery of their deficit.

CONCLUSION. Computerized dynamic posturography has a particularly important role in the evaluation, monitoring and rehabilitation of the patients with peripheral vestibular deficiency.

KEYWORDS: computerized dynamic posturography, vestibular rehabilitation, peripheral vestibular disorders, foam platform

\section{INTRODUCTION}

Computerized dynamic posturography (CDP) is a quantitative method which evaluates the ability of the human body to maintain balance in upright position in various testing conditions that replicate real-life situations ${ }^{1}$.

Body position, in relation to gravity and environmental landmarks, is perceived by various information coming from the proprioceptive, vestibular and visual systems. The visual system is important in maintaining balance when the environment is in motion ${ }^{2}$. For maintaining balance, the patient must coordinate eye movements to obtain a stable image of the environment on the retina. Maintaining balance during movement is achieved with the help of the proprioceptive system that provides information from the proprioceptive receptors in the striated muscles of the legs and neck $^{3}$. The role of the vestibular system becomes particularly important when the first two systems do not work properly ${ }^{4}$. In addition to assessing and identifying patients with peripheral vestibular deficit, CDP can also be used in vestibular rehabilitation (VR) programs ${ }^{5}$. VR helps regaining postural control and elim- 
inates the risks of falls in patients with vestibular deficiency.

The role of VR program is to interconnect the information received from the somatosensory and visual system with those provided by the vestibular system ${ }^{6-8}$.

Our study aimed to assess the role of CDP in the VR of patients suffering from peripheral vestibular syndrome.

\section{MATERIAL AND METHODS}

\section{Patients}

We conducted a prospective cohort study during January 2012 - December 2014 that analyzed 30 patients with unilateral peripheral vestibular deficiency syndrome who received vestibular rehabilitation using platform posturography ${ }^{9}$. All patients included in the study signed an informed consent form to participate in the study. All patients have completed the vestibular rehabilitation program on platform posturography.

Inclusion criteria: patients who have never benefited from VR on posturography platform and patients with normal neurological exam. We considered also the patients with benign paroxysmal positional vertigo (BPPV) that previously benefited from canalith repositioning procedures, but still showed unsteadiness evidenced by posturography.

We excluded the patients who refused participation in the study, those with associated neurological disorders (e.g. Dementia, Parkinson's disease), psychiatric disorders, visual disturbances; patients treated with neuroleptics, sedatives, antiepileptics, antidepressants, drug or alcohol abuse, patients with severe orthopaedic dysfunction and arthrosis of the ankle, hip and knee.

\section{Study Protocol}

In this study we used the Posturographic Synapsys System (version 3.0, SYNAPSYS, Marseille, France). All the patients were evaluated using the Sensory Organization Test that monitors the three sensory systems involved in maintaining balance (proprioceptive, visual and vestibular). The evaluation was conducted at the onset and at the end of eight sessions of VR. In our study, we considered the results obtained from patients that were tested with eyes open (EO) and eyes closed (EC) on foam platform. Each test consisted of two trials that lasted 20 seconds each and the VR program consisted in 8 sessions ( 2 sessions/week) ${ }^{10,11}$. The VR program consisted in rehabilitation games included in the software used by the posturographic system. Each session began with a test reference which helped monitor the patient's evolution throughout the rehabilitation. The reference game was the same for each session and patient included in the study. In the rehabilitation session we included games from four groups: Stabilization, Weight shift, Weight bearing and Postural control.

\section{Statistical analysis}

The collected data was analyzed using the software Stata IC 11 (StataCorp. 2009. Stata: Release 11 Statistical Software. College Station, TX, USA). The data was expressed as a percentage, frequency, mean, standard deviation, depending on the situation. The $T$ test (Student) was used for the evaluation of quantitative differences in the calculation of media. The level of statistical significance was set at 0.05 .

The normal values of parameters followed during the study: Romberg coefficient, statokinesigram (SKG), maximum amplitude of the degree of deviation in anterior/posterior (AP) and medial/lateral (ML) planes were obtained from the posturographic system software (Table 1).

The normal values used for the Romberg coefficient were between 85-241.

To evaluate the therapeutic success of vestibular rehabilitation on platform posturography, we based our observations on the following ranges of values for each parameter obtained on foam platform after the 8 sessions of VR (Table 2).

These ranges of values were used to be able to classify patients included in the study, according to their performance in two groups: stationary and improved. The creation of these groups was necessary to assess the therapeutic success of vestibular rehabilitation on platform posturography in patients with unilateral peripheral vestibular deficiency syndrome.

Table 1

Normal parameters values for foam platform

\begin{tabular}{cccc}
\hline Foam & SKG (mm2) & Max amplitude AP (mm) & Max amplitude ML (mm) \\
\hline Eyes open & $437-618$ & 37.19 & 39.99 \\
\hline Eyes closed & $909-1325$ & 66.72 & 58 \\
\hline
\end{tabular}


Table 2

Reference values for improved and stationary patient groups

\begin{tabular}{ccc}
\hline Foam & IMPROVED & STATIONARY \\
\hline SKG eyes open $(\mathrm{mm} 2)$ & $618-900$ & $>900$ \\
\hline AP $(\mathrm{mm})$ & $38-50$ & $>50$ \\
\hline ML (mm) & $40-52$ & $>52$ \\
\hline SKG eyes closed (mm2) & $1325-2200$ & $>2200$ \\
\hline AP $(\mathrm{mm})$ & $67-80$ & $>80$ \\
\hline$M L(\mathrm{~mm})$ & $58-70$ & $>70$ \\
\hline
\end{tabular}

\section{RESULTS}

The clinical study was conducted on a group of 30 patients with unilateral peripheral vestibular deficiency syndrome, including 14 women $(46.67 \%)$ and 16 men $(53.33 \%)$; 13 cases originated from rural areas $(43.33 \%)$ and 17 cases from urban areas $(56.67 \%)$. The mean age was 55.8 years \pm 12.12 SD (age between 33 and 78 years).

We analysed the associated risk factors and we encountered 10 patients with cervical spondylosis $(33.33 \%), 8$ cases with systemic hypertension $(26.67 \%)$, 8 cases with dyslipidemia $(26.67 \%), 5$ cases $(16.67 \%)$ with carotid atheromatosis and 5 cases $(16.67 \%)$ with diabetes mellitus. Vestibular rehabilitation therapy (VR) on posturography platform was performed to patients with unilateral peripheral vestibular deficiency syndrome (vestibular neuronitis, Meniere's syndrome) and BPPV patients presenting with postural instability (previously benefited from canalith repositioning procedures). In this study we did not include patients diagnosed with acoustic neurinoma.

We recorded statistically significant differences between the results obtained on platform posturography before (RQ1, SKG1, AP1, ML1) and after (RQ2, SKG2, AP2, ML2) vestibular rehabilitation, especially with EO and EC on foam platform $(\mathrm{p}<0.05)$, as one can see in Table 4.

Statistical analysis of parameters with EO and EC on foam platform before and after vestibular rehabilitation on platform posturography

In EO condition, there was a significant decrease of the statokinesigram after VR $(\mathrm{p}<0.05)$ from an average of $923.61 \pm 805.86 \mathrm{~mm}$ before VR to an average of $552.366 \pm 456.885$ after VR. The average difference was 371.2 ( $\mathrm{p}=0.0002$; Student t-test; $95 \% \mathrm{CI}$ varies between 195.55 and 545.92). After the rehabilitation, we observed a decrease in balance on AP plan $(\mathrm{p}<0.05)$ from an average of $37.53 \pm 13.87 \mathrm{~mm}$ to an average of $29.40 \pm 7.97 \mathrm{~mm}$. The average difference was equal to $8.13(\mathrm{p}=0.0081$; Student t-test; $95 \% \mathrm{CI}=$ 2.2 - 13.9). Regarding balance on ML plan after VR, the results show a significant decrease $(p<0.05)$ from an average of $43.27 \pm 22.62 \mathrm{~mm}$ to $17.92 \mathrm{~mm} \pm 31.09$ $\mathrm{mm}$ after the eight VR sessions. The average difference was $12.18(\mathrm{p}<0.0001$; Student t-test; 95\% CI $=7.6$ 16.67).

Table 3

Distribution of cases according to etiology

\begin{tabular}{cclcc}
\hline & Vestibular neuronitis & $\begin{array}{l}\text { Unknown cause for } \\
\text { vestibular syndrome }\end{array}$ & Meniere syndrome & BPPV* \\
\hline Number of cases & 12 & 7 & $23.33 \%$ & 4 \\
\hline$\%$ & $40 \%$ & $23.33 \%$ & $13.33 \%$ \\
\hline
\end{tabular}

* For the study we selected BPPV patients with postural instability after canalith repositioning procedure. Dix-Hallpike maneuver used forthe posterior semicircular canals and specific manoeuvres for the lateral semicircular canals were negative for these patients. 
Table 4

Correlations of mean values of the studied parameters

\begin{tabular}{|c|c|c|c|c|c|}
\hline Variable & Mean & Std. Dev. & Min. & Max. & p \\
\hline RQ1 & 161.93 & 96.01 & 56 & 533 & 0.8691 \\
\hline $\mathrm{RQ2}$ & 166 & 108.73 & 44 & 642 & \\
\hline SKG1 foam EO & 923.61 & 805.86 & 358.17 & 4782.95 & 0.0002 \\
\hline SKG2 foam EO & 552.366 & 456.885 & 171.98 & 2800 & \\
\hline AP1 foam E0 & 37.53 & 13.87 & 22.9 & 99.8 & 0.0081 \\
\hline AP2 foam E0 & 29.40 & 7.97 & 16.6 & 44.4 & \\
\hline ML1 foam E0 & 43.27 & 22.62 & 18.8 & 146.1 & $<0.0001$ \\
\hline ML2 foam E0 & 31.09 & 17.92 & 17.4 & 120 & \\
\hline SKG1 foam EC & 43.27 & 22.62 & 18.8 & 146.1 & $<0.0001$ \\
\hline SKG2 foam EC & 31.09 & 17.92 & 17.4 & 120 & \\
\hline AP1 foam EC & 71.52 & 20.69 & 40.1 & 116.7 & $<0.0001$ \\
\hline AP2 foam EC & 47.40 & 9.42 & 30.2 & 64.2 & \\
\hline ML1 foam EC & 67.88 & 21.83 & 35.6 & 140.3 & $<0.0001$ \\
\hline ML2 foam EC & 38.65 & 13.99 & 23.9 & 90.2 & \\
\hline SKG1 before VR & 15.06 & 3,47 & 10 & 22 & $<0.0001$ \\
\hline SKG2 after VR & 5.43 & 1.67 & 2 & 10 & \\
\hline T1 before VR & 31.11 & 4.53 & 20.32 & 38.42 & $<0.0001$ \\
\hline T2 after VR & 15.14 & 1.55 & 12.32 & 18.2 & \\
\hline
\end{tabular}

*SKG1 and SKG2 before and after VR: statokinesigram of the reference game before and after vestibular rehabilitation process; $\mathrm{T} 1$ and T2 before and after VR: the time interval (seconds) of the reference game before and after vestibular rehabilitation process

In EC condition, there was a significant decrease of the statokinesigram after $\mathrm{RV}(\mathrm{p}<0.05)$ from an average of $2443.43 \pm 947.90 \mathrm{~mm}$ before VR to an average of $1142.99 \pm 641.53 \mathrm{~mm}$ after VR. The average difference was of $1300.44(p<0.0001$; Student t-test; 95\% CI between 1018.5 and 1582.3). After VR, we observed a decrease in balance on AP plan $(\mathrm{p}<0.05)$ from the average value of $71.52 \pm 20.69 \mathrm{~mm}$ before to an average of $47.40 \pm 9.42 \mathrm{~mm}$. The average difference is 24.11 ( $\mathrm{p}<0.0001$; Student t-test; 95\%CI 16.8 to 31.3 is from $)$.
Regarding ML balance, results after VR showed a significant decrease $(\mathrm{p}<0.05)$ from $67.88 \pm 21.83 \mathrm{~mm}$ to an average of $38.65 \pm 13.99 \mathrm{~mm}$; the average difference of 29.23 ( $\mathrm{p}<0.0001$; Student t-test; 95\% CI was between 23 and 35.4$)$.

Statistical analysis of statokinesigram (cm2) and time (seconds) of the reference game before and after the vestibular rehabilitation process

In terms of the SKG obtained in the reference game 
after VR, there was a significant decrease $(\mathrm{p}<0.05)$ from an average of $15.06 \pm 3.47 \mathrm{~cm} 2$ before VR to an average of $5.43 \pm 1.67 \mathrm{~cm} 2$ after VR. The average difference was $9.63(\mathrm{p}<0.0001$; Student t-test; $95 \%$ CI between 8.58 and 10.67). After VR, the time interval of the reference game $(p<0.05)$ decreased from the mean value $31.11 \pm 4.5 \mathrm{~s}$ before VR to a mean value of $15.14 \pm 1.55 \mathrm{~s}$. The average difference was equal to 15.97 ( $\mathrm{p}<0.0001$; Student t-test; 95\% CI = 14.57 -17.37).

In both cases there was a significant decrease in parameters values after VR $(\mathrm{p}<0.05)$.

\section{Statistical analysis of the Romberg coefficient be- fore and after vestibular rehabilitation on platform posturography}

Comparing the final mean values to the initial mean values of the Romberg coefficient (RQ2 versus RQ1), we found a mean difference of $4.066(\mathrm{p}=0.8691$; Student t-test; $95 \%$ CI ranging between 54 and 49.94). Given that $p>0.05$, the difference between the two values is not statistically significant.

The final results showed that of the 30 patients with unilateral peripheral vestibular deficiency 28 (93.33\%) were fully rehabilitated, 1 patient showed improvement $(3.33 \%)$ and 1 patient was stationary $(3.33 \%)$. It should be noted that changes were evident since the end of the first session.

\section{DISCUSSIONS}

Computerized dynamic posturography (CDP) is a technique commonly used to quantify postural control. In addition to assessing and identifying patients with vestibular deficit, CDP can be used during VR sessions $^{12-14}$. Although this technique is commonly used, there are few studies that demonstrate its effectiveness in using the platform posturography for VR of patients with unilateral peripheral vestibular deficiency ${ }^{15,16}$.

Nardone has highlighted the effectiveness of vestibular rehabilitation on the platform in an important study conducted in 2010 on 33 patients with peripheral vestibular deficit ${ }^{17}$. Furthermore, studies in patients with central vestibular deficit showed that vestibular rehabilitation with platform posturography can significantly decrease the risk of falls, improve postural stability and increase the quality of life of these patients $^{18,19}$.

Our results showed that patients had improved postural control after using the VR on posturography platform. On average, patients with peripheral vestibular disease with unilateral deficit, showed statistically significant changes $(p<0.05)$ in the values of the six parameters studied. The statokinesigram and the time of the reference game before and after VR improved significantly $(\mathrm{p}<0.0001)$, proving the therapeutic success of VR in the patients included in our study. At the end of the rehabilitation program, the results were more than satisfactory; $93.33 \%$ of cases achieved full recovery of the vestibular function.

Even though the recovery of the vestibular function depends on the severity of vestibular damage, the studies show that controlled and monitored rehabilitation programs can lead to a high level of recovery ${ }^{20,21}$.

\section{CONCLUSIONS}

CDP allows evaluation and rehabilitation of vestibular function by assessing the overall balance and identification of the systems used by the patient to maintain posture. Using platform posturography in vestibular rehabilitation programs allows multisensory rehabilitation by using the interconnection between visual, vestibular and proprioceptive information.

Given the encouraging results of the present study, we can say that the monitored parameters in this article may be useful for creating guidelines for vestibular rehabilitation programs in the future.

Conflict of interests: None declared.

Financial support: None declared.

\section{REFERENCES}

1. Furman J.M. - Posturography: uses and limitations. Baillieres Clin Neurol., 1994;3(3):501-513.

2. Mirka A., Black F.O. - Clinical application of dynamic posturography for evaluating sensory integration and vestibular dysfunction. Neurol Clin., 1990;8(2):351-359.

3. Furman J.M. - Role of posturography in the management of vestibular patients. Otolaryngol Head Neck Surg., 1995;112(1):8-15.

4. Luchikhin L.A. - Posturography: efficacy and prospects in Otorhinolaryngology. Vestn Otorinolaringol., 1997;(1):19-23.

5. Weber P.C. , Cass S.P. - Clinical assessment of postural stability. Am J Otol., 1993;14(6):566-569

6. Cohen H.S., Kimball K.T. - Increased independence and decreased vertigo after vestibular rehabilitation. Otolaryngol Head Neck Surg., 2003;128(1):60-70.

7. Tran Ba Huy P. - Schwannoma and other CPA lesions. Barcelona. Ed. E Garcia-Ibanez, Medimond, 2007;pp.105-110.

8. Mbongo F., Qu'hen C., Vidal P.P., Tran Ba Huy P., Waele C. - Role of vestibular input in triggering and modulating postural responses in unilateral and bilateral vestibular loss patients. Audiol Neurootol., 2009;14(2):130-138. doi: 10.1159/000162665.

9. Shepard N.T., Telian S.A., Smith-Wheelock M., Raj A. - Vestibular and balance rehabilitation therapy. Ann Otol Rhinol Laryngol., 1993;102:198205.

10. Asai M., Watanabe Y., Ohashi N., Mizukoshi K. - Evaluation of vestibular function by dynamic posturography and other equilibrium examinations. Acta Otolaryngol Suppl., 1993;504:120-124.

11. Curthoys I.S., Halmagyi M. - Vestibular compensation: clinical changes in vestibular function with time after unilateral vestibular loss. In: Herdman 
S.J. - Vestibular Rehabilitation. 3rd ed. Philadelphia: F.A. Davis Co., 2007;pp.172-194.

12. Shumway-Cook A., Horak F.B., Yardley L., Bronstein A.M. Rehabilita $\neg$ tion of balance disorders in the patient with vestibular pathology. In: Bronstein A.M., Brandt T., Woollacott M. - Clinical Disorders of Balance Posture and Gait. London: Arnold, 1996;pp.211-235.

13. Horak F.B. - Postural compensation for vestibular loss and implications for rehabilitation. Restor Neurol Neurosci., 2010;28(1):57-68. doi: 10.3233/RNN-2010-0515.

14. Brandt T., Dieterich M. - Postural imbalance in peripheral and central vestibular disorders. In: Bronstein A.M., Brandt T., Woollacott M.H., Nutt J.G. - Clinical Disorders of Balance, Posture and Gait. 2nd ed. London: Arnold, 2004;pp.146-162.

15. Badaracco C., Labini F.S., Meli A., De Angelis E., Tufarelli D. - Vestibular rehabilittion outcomes in chronic vertiginous patients through computerized dynamic visual acuity and Gaze stabilization test. Otol Neurotol., 2007;28(6):809-813.

16. Cakrt O., Chovanec M., Funda T.., et al. - Exercise with visual feedback improves postural stability after vestibular schwannoma surgery. Eur Arch
Otorhinolaryngol., 2010;267(9):1355-1360. doi: 10.1007/s00405-0101227-x.

17. Nardone A., Godi M., Artuso A., Schieppati M. - Balance rehabilitation by moving platform and exercises in patients with neuropathy or vestibular deficit. Arch Phys Med Rehabil., 2010;91(12):1869-1877. doi: 10.1016/j. apmr.2010.09.011.

18. Rossi-Isquierdo M., Soto-Varela A., Santos-Perez S., et al. - Vestibular rehabilitation with computerised dynamic posturography in patients with Parkinson's disease: improving balance impairment. Disab Rehabil., 2009;31 (23):1907-1916. doi: 10.1080/09638280902846384.

19. Musat G. - Computerized posturography in the diagnosis and treatment of the instability of the elderly. Romanian Journal of Neurology, 2013;12(2):58-62.

20. Black F.O., Angel C.R., Pesznecker S.C., Gianna C. - Outcomes analysis of individualized vestibular rehabilitation protocols. Am J Otol., 2000;21(4):543-551.

21. Badke M.B., Miedaner J.A., Shea T.A., Grove C.R., Pyle G.M. - Effects of vestibular and balance rehabilitation on sensory organization and dizziness handicap. Ann Otol Rhinol Laryngol., 2005;114(1 Pt 1):48-54. 